\title{
FINITELY GENERATED GROUPS, $p$-ADIC ANALYTIC GROUPS, AND POINCARÉ SERIES
}

\author{
MARCUS P. F. DU SAUTOY
}

\section{INTRODUCTION}

Igusa [I 1, I 2] was the first to exploit $p$-adic integration with respect to the Haar measure on $\mathbf{Q}_{p}$ in the study of Poincare series arising in number theory and developed a method using Hironaka's resolution of singularities to evaluate a limited class of such integrals. Denef [D 1, D 2] and, more recently, Denef and van den Dries [DvdD] have applied results from logic, profiting from the flexibility of the concept of definable, greatly to enlarge the class of integrals amenable to Igusa's method. In [DvdD] these results are employed to answer questions posed by Serre [S] and Oesterlé $[\mathrm{O}]$ concerning the rationality of various Poincare series associated with the $p$-adic points of a closed analytic subset of $\mathbf{Z}_{p}^{m}$. In this note we apply these techniques to prove that various Poincare series associated with finitely generated groups and $p$-adic analytic groups are rational in $p^{-s}$, extending results of [GSS].

\section{RESULTS}

Let $G$ be a group and denote by $a_{n}(G)$ the number of subgroups of index $n$ in $G$. We are interested in groups for which $a_{n}(G)$ is finite for every $n \in \mathbf{N}$. For each prime $p$, we can then associate the following Poincare series with this arithmetical function:

$$
\zeta_{G, p}(s)=\sum_{n=0}^{\infty} a_{p^{n}}(G) p^{-n s}=\sum_{H \in \mathbf{X}_{p}}|G: H|^{-s}
$$

where $\mathbf{X}_{p}=\{H \leq G: H$ has finite $p$-power index in $G\}$.

Received by the editors June 22, 1989 and, in revised form, November 18 , 1989.

1980 Mathematics Subject Classification (1985 Revision). Primary 22E20; Secondary $03 \mathrm{C} 10,11 \mathrm{M} 41,20 \mathrm{E} 18,20 \mathrm{~F} 99$. 


\section{§1. $p$-Adic ANALYTIC GROUPS}

We consider firstly the case where $G$ is a compact $p$-adic analytic group-that is, a compact topological group with the underlying structure of a $p$-adic analytic manifold with respect to which the group operations are analytic (see [Lz] and [DduSMS]). For such groups, $a_{n}(G)$ is finite for every $n$. We wish to announce the following:

Theorem 1. If $G$ is a compact p-adic analytic group, then $\zeta_{G, p}(s)$ is rational in $p^{-s}$.

The philosophy behind the proof is to express our Poincare series as a $p$-adic integral

$$
\int_{M}|f(\mathbf{x})|^{s}|g(\mathbf{x})||d \mathbf{x}|
$$

where $|d \mathbf{x}|$ is the normalized Haar measure on $\mathbf{Z}_{p}^{n}$ and the functions $f, g: \mathbf{Z}_{p}^{n} \rightarrow \mathbf{Z}_{p}$ and the subset $M$ are definable in the language describing the analytic theory of the $p$-adic numbers. We can then evaluate such definable integrals applying the techniques developed by Denef and van den Dries [DvdD] (which include quantifier elimination results for the analytic theory of $\mathbf{Z}_{p}$ ) to prove our theorem.

The translation from our Poincare series to such a definable $p$-adic integral makes full use of Lazard's results on the close relationship between the structure of compact $p$-adic analytic groups and filtrations defined on such groups [Lz]. In answer to "Hilbert's 5 th problem for $p$-adic analytic groups," Lazard has shown that a compact topological group has the structure of a $p$-adic analytic group if and only if there exists a normal subgroup $G_{1}$ of finite index in $G$ which is $p$-saturable-that is, there exists a filtration on $G_{1}$

$$
G_{1}>G_{2}>\cdots>G_{i}>\cdots
$$

such that: (i) $G_{1}$ is a pro- $p$ group with a fundamental system of neighborhoods of the identity given by $\left\{G_{i}: i \in \mathbf{N}\right\}$; (ii) for all $i \geq 1, G_{i} / G_{i+1}$ is an elementary Abelian $p$-group of finite rank; and (iii) for all $i \geq 1$, the map $P_{i}: G_{i} / G_{i+1} \rightarrow G_{i+1} / G_{i+2}$ defined by $x G_{i+1} \rightarrow x^{p} G_{i+2}$ is an isomorphism of $\mathbf{F}_{p}$ vector spaces.

A $p$-saturable group has the underlying structure of a pro- $p$, $p$-adic analytic group with a global coordinate system $\mathbf{Z}_{p}^{r}$ given by $p$-adic powers of elements $x_{1}, \ldots, x_{r}$ where $x_{1} G_{2}, \ldots, x_{r} G_{2}$ is an $\mathbf{F}_{p}$ vector space basis for $G_{1} / G_{2}$. 
We first prove Theorem 1 in the case where $G$ is a $p$-saturable group. Recall that subgroups of finite index in a pro- $p$ group have $p$-power index. The idea is to associate with every subgroup $H$ of finite index in $G$, a subset $M(H)$ of $r \times r$ matrices over $\mathbf{Z}_{p}$ whose rows form coordinates for a good basis for $H$. Every subgroup of finite index in a compact $p$-adic analytic group is open. So, there exists $m$ such that $H \geq G_{m}$. We define the concept of a good basis for $H$ as a set of elements $h_{1}, \ldots, h_{r}$ such that, for each $i=1, \ldots, m$, if we let $v_{j}=h_{j}^{p^{(i, j)}} \in H \cap G_{i}$ for $\omega\left(h_{j}\right) \leq i$, where $e(i, j)=i-\omega\left(h_{j}\right)$ and $\omega(g)=n$ if $g \in G_{n} \backslash G_{n+1}$, then

$$
\left\{v_{j} G_{i+1}: j \text { such that } \omega\left(h_{j}\right) \leq i\right\}
$$

is an $\mathbf{F}_{p}$ vector space basis for $\left(H \cap G_{i}\right) G_{i+1} / G_{i+1}$. The index of $H$ in $G$ is encoded in the measure of the subset $M(H)$ and we identify functions $f, g: \mathbf{Z}_{p}^{n} \rightarrow \mathbf{Z}_{p}$ such that, for all subgroups $H$ of finite index in $G$

$$
|G: H|^{-s}=\int_{M(H)}|f(\mathbf{x})|^{s}|g(\mathbf{x})||d \mathbf{x}| .
$$

Summing over all subgroups of finite (necessarily $p$-power) index in $G$, we can express our Poincaré series $\zeta_{G, p}(s)$ as a $p$-adic integral of the form (2) where $M \subseteq \mathbf{Z}_{p}^{r^{2}}$ is the (disjoint) union of subsets $M(H)$ for all $H \in \mathbf{X}_{p}$.

The problem now is to show that this integral is definable in the sense of [DvdD]. The set of $r$-tuples of elements of $G$ which form a good basis for some subgroup of finite index in $G$ is definable by a filtered group theoretic statement. We show how to translate such statements into statements about coordinates of elements of $G$ definable now in the language describing the analytic theory of the $p$-adic numbers. Using this translation we can show that the subset $M$ is definable. With regards to the functions $f$ and $g$, we show that there exists a finite partition of $\mathbf{Z}_{p}^{r^{2}}$ into definable subsets such that, on each subset, $f$ and $g$ are defined by polynomial functions. Thus $f$ and $g$ are definable functions. We are then in a position to apply the techniques of [DvdD] to this definable integral and thus prove Theorem 1 in the case where $G$ is a $p$ saturable group.

We extend this to a proof of Theorem 1 using the following ideas. Let $G$ be a compact $p$-adic analytic group and $G_{1}$ a normal $p$-saturable subgroup of finite index in $G$. If $H$ is subgroup of 
$p$-power index in $G$, then it is determined by $H_{1}=H \cap G_{1}$ and a transversal for $H_{1}$ in $H$. We associate with each $H$ a subset $N(H)$ consisting of coordinates both for a good basis for $H_{1}$ and coordinates for a transversal for $H_{1}$ in $H$. Extending our integral associated with the $p$-saturable group $G_{1}$, we can express $\zeta_{G, p}(s)$ as a definable $p$-adic integral over the union of the subsets $N(H)$. We can therefore apply [DvdD] to prove that our Poincare series associated with the compact $p$-adic analytic group $G$ is rational in $p^{-s}$.

\section{§2. Finitely GENERATED GROUPS}

Theorem 1 has various corollaries for finitely generated groups. If $\Gamma$ is a finitely generated group, then $a_{n}(\Gamma)$ is finite for all $n$. We can therefore consider the Poincare series defined in (1). We consider first a variant of this Poincaré series. Define $a_{n}^{\triangleleft \triangleleft}(\Gamma)$ to be the number of subnormal subgroups of index $n$ in $\Gamma$. For each prime $p$, we associate with the finitely generated group $\Gamma$, the following Poincaré series:

$$
\zeta_{\Gamma, p}^{\triangleleft \triangleleft}(s)=\sum_{n=0}^{\infty} a_{p^{n}}^{\triangleleft \triangleleft}(\Gamma) p^{-n s}=\sum_{H \in \mathbf{Y}_{p}}|\Gamma: H|^{-s},
$$

where $\mathbf{Y}_{p}=\{H \leq \Gamma: H$ is subnormal of $p$-power index in $\Gamma\}$.

We say that $a_{p^{n}}(\Gamma)$ grows polynomially if there exists $c \in \mathbf{N}$ such that $a_{p^{n}}(\Gamma) \leq p^{n c}$ for all $n$. Similarly for $a_{p^{n}}^{\triangleleft \triangleleft}(\Gamma)$. We then have the following:

Theorem 2. Let $\Gamma$ be a finitely generated group and $p$ a prime. If $a_{p^{n}}^{\triangleleft \triangleleft}(\Gamma)$ grows polynomially, then $\zeta_{\Gamma, p}^{\triangleleft \triangleleft}(s)$ is rational in $p^{-s}$.

There is a one-to-one correspondence between subnormal subgroups of finite $p$-power index in $\Gamma$ and subgroups of finite index in the pro- $p$ completion $G$ of $\Gamma$. So $\zeta_{G, p}(s)=\zeta_{\Gamma, p}^{\triangleleft \triangleleft}(s)$. By Lubotzky and Mann's characterization of pro- $p$ groups which have the underlying structure of a $p$-adic analytic group [LM], if $a_{p^{n}}(G)=a_{p^{n}}^{\triangleleft \triangleleft}(\Gamma)$ grows polynomially, then $G$ is a $p$-adic analytic pro- $p$ group. By Theorem $1, \zeta_{\Gamma, p}^{\triangleleft \triangleleft}(s)$ is rational in $p^{-s}$.

We recall the definition of an upper p-chief factor of $\Gamma$-that is, a chief factor of some finite quotient of $\Gamma$ whose order is divisible by $p$. We then have the following:

Theorem 3. Let $\Gamma$ be a finitely generated group and $p$ a prime such that the order of all p-chief factors of $\Gamma$ is bounded. If $a_{p^{n}}(\Gamma)$ grows polynomially, then $\zeta_{\Gamma, p}(s)$ is rational in $p^{-s}$. 
The bound on the order of $p$-chief factors in $\Gamma$ implies that there exists a normal subgroup $\Gamma_{0}$ of finite index in $\Gamma$ whose subgroups of $p$-power index are all subnormal. We construct a finite extension $G$ of the pro- $p$ completion of $\Gamma_{0}$ whose subgroups of finite index are in one-to-one correspondence with subgroups of $p$-power index in $\Gamma$. If $a_{p^{n}}(\Gamma)$ grows polynomially, then, by $[\mathrm{LM}], G$ is a finite extension of a $p$-adic analytic pro- $p$ group. So $G$ is a compact $p$-adic analytic group and by Theorem $1, \zeta_{G, p}(s)=\zeta_{\Gamma, p}(s)$ is rational in $p^{-s}$.

Theorem 3 includes a large class of examples, some of which we collect together in the following corollary. We recall that the upper p-rank of $\Gamma$ is the supremum of $r(P)$ as $P$ ranges over all $p$-subgroups of finite quotients of $\Gamma$, where $r(P)$ is the rank of $P$.

Corollary 4. If $\Gamma$ is a finitely generated group of finite upper prank, then $\zeta_{\Gamma, p}(s)$ is rational in $p^{-s}$.

This follows from a remark in [MS].

\section{REFERENCES}

[D 1] J. Denef, The rationality of the Poincare series associated to the p-adic points on a variety, Invent. Math. 77 (1984), 1-23.

[D 2] _ On the evaluation of certain p-adic integrals, Seminaire de Théorie des Nombres, Paris 1983-4, Progr. Math. 59 (1985), 25-47.

[DvdD] J. Denef and L. van den Dries, P-adic and real subanalytic sets, Ann. of Math. 128 (1988), 79-138.

[DduSMS] J. Dixon, M. P. F. du Sautoy, A. Mann and D. Segal, Analytic pro-p groups, London Math. Society Lecture Notes (to appear).

[GSS] F. J. Grunewald, D. Segal, and G. C. Smith, Subgroups of finite index in nilpotent groups, Invent. Math. 93 (1988), 185-223.

[I 1] J.-I. Igusa, Complex powers and asymptotic expansions I, J. Reine Angew. Math. 268/269 (1974), 110-130.

[I 2] _ Some observations on higher degree characters, Amer. J. Math. 99 (1977), 393-417.

[Lz] M. Lazard, Groupes analytiques p-adiques, Publ. Math. Inst. Hautes Ėtudes Sci. 26 (1965), 389-603.

[LM] A. Lubotzky and A. Mann, On groups of polynomial subgroup growth, Invent. Math. (to appear).

[MS] A. Mann and D. Segal, Uniform finiteness conditions in residually finite groups, Proc. London Math. Soc. (to appear). 
[O] J. Oesterlé, Réduction modulo $p^{n}$ des sous-ensembles analytiques fermés de $\mathrm{Z}_{p}^{N}$, Invent. Math. 66 (1982), 325-341.

[S] J.-P. Serre, Quelques applications du théorème de densité de Chebotarev, Publ. Math. Inst. Hautes Ėtudes Sci. 54 (1981), 123-202.

Mathematical Institute, 24-29 St. Giles, Oxford, England

Current address: School of Mathematical Sciences, Queen Mary and Westfield College, University of London, London E1 4N5, England 